\title{
Design of Mine Locomotive System Based on CAN Bus
}

\author{
Yuanhong Li, Quanzhu Zhang and Wenshan Zhang \\ North China Institute of Science and Technology, Information and Control Technology Institute \\ Beijing East Yanjiao 101601
}

\begin{abstract}
Based on CAN bus, this paper studies the system control and management system of locomotive in mine, analyzes the working principle of locomotive system, gives the CAN bus scheme, hardware circuit design and CAN communication protocol,and implements long-distance, high-reliability communication function and remote monitoring function. Experiments show that the auxiliary system based on CAN bus control easier, operation more secure, as well as improving the control performance and service life of the electric locomotive .
\end{abstract}

\section{Introduction}

With the development of power electronics technology, CAN bus technology to use, coal mine electric locomotive now the signal transmission system can't meet the requirements of the development of the times ${ }^{[1]}$.Electric locomotive now the auxiliary converter power supply has some shortcomings: Each device on the locomotive (transformers, air conditioning units, etc.) must individually lead a line to control the start and stop of the device, failure and all the feedback of the signal, and transmitted to the console, and automatic or manual control.As shown in Figure 1, the system for the auxiliary converter working principle, various devices, contactors, etc,with the key switch to control, then this is not only complicated wiring, maintenance inconvenience, the most important is still very easy to cause dangerous accidents.But we use the auxiliary converter system instead of the original system, combined with CAN bus technology, it is very convenient to achieve the above control, both to improve the efficiency, while the system also take into account advanced science and technology, and has a good use of value, there is a broad application market ${ }^{[2-3]}$.

\section{The overall structure of the system}

Figure 1 shows a block diagram of the system-assisted converter, which is composed of phase-controlled

rectifiers, four inverters, and various electrical equipment (brake fan, traction machine, compressor, oil pump).And each device is controlled by a contactor (and a spare contactor). This system will first AC794 voltage through the phase-controlled rectifier into DC540V, and then
DC540V voltage inverter, inverted into AC380V supply equipment needed to use the device, in order to ensure normal operation of equipment.

In conjunction with Figure 1, we draw the CAN bus block diagram.As shown in picture 2,there are the start stop button and the display screen of each device on the driver's console, which can display the real-time status of each device.Before starting, the driver console receives phase fault rectifier fault signal and 4 inverter fault signal, if there is no fault, then start the phase-controlled rectifier, and then as needed to open the various inverter operation.

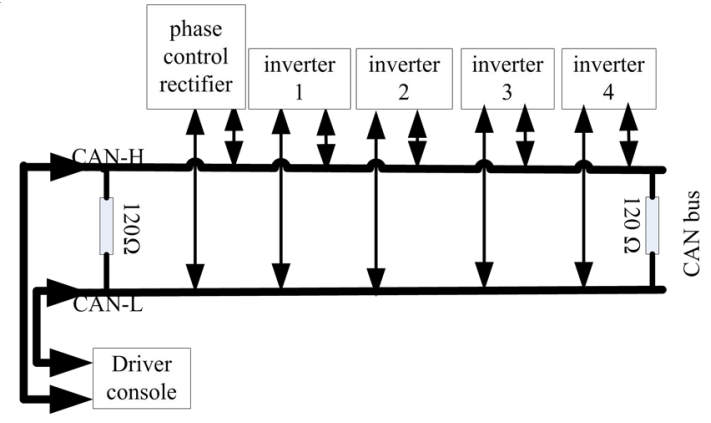

Figure 2 CAN bus system architecture

\section{Hardware design}

Figure 3 shows the CAN circuit block diagram, which phase control rectifier, four inverters are used a STM32F103ZET6 chip to control.STM32F103 chip comes with CAN controller used to achieve data transmission.bxCAN is STM32F1 comes with CAN controller, which supports CAN protocol 2.0A and CAN2.0B.This controller uses the smallest CPU load to 
handle a large number of received packets, but also supports the priority of packet transmission ${ }^{[4]}$.

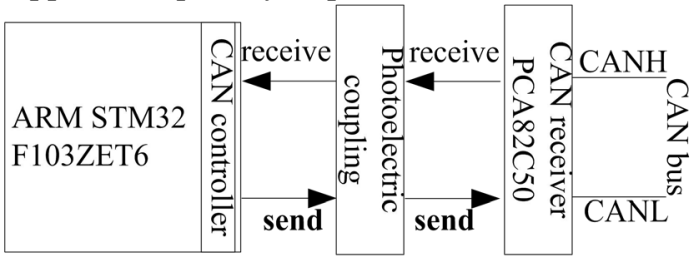

Figure 3 CAN circuit block diagram

\subsection{CAN circuit design}

Figure 4 for the separate power supply module, the use of "F0505D-1W" DC-DC converter, The 5V voltage is converted into a $5 \mathrm{~V}$ power isolation, improve the noise immunity of the bus, isolated power supply CAN optocoupler isolation and transceiver , and increased CAN bus stability and anti-jamming capability.

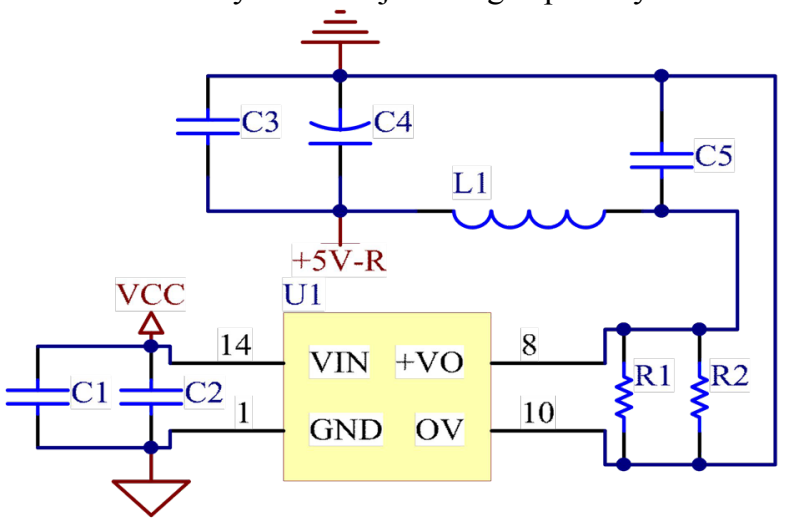

Figure 4 power supply module

Figure5 shows the optical isolation circuit, we use high-speed optocoupler 6N137, the chip sent by the signal CANTXD and received signal CANR, with optocoupler isolation, so that the speed of the CAN network can be achieved and the speed of the previous network drive the same,so that the signal transmission more timely, stable, reliable, the most important can improve the anti-jamming.

Figure 6 shows the CAN transceiver circuit, we use the PCA82C50 chip, the chip can provide differential transmission capacity of the bus, CAN controller to provide differential reception capability, and the chip to CAN in high-speed mode, so that the transmitter output stage transistors at the fastest possible opening and closing speed, improve the efficiency of the CAN. The chip in the circuit accepts the CANT, CANR signal from the optocoupler, will become a stable differential signal, after the follow-up filter circuit, the protection circuit sent to the CAN bus, which can improve the signal anti-jamming capability.
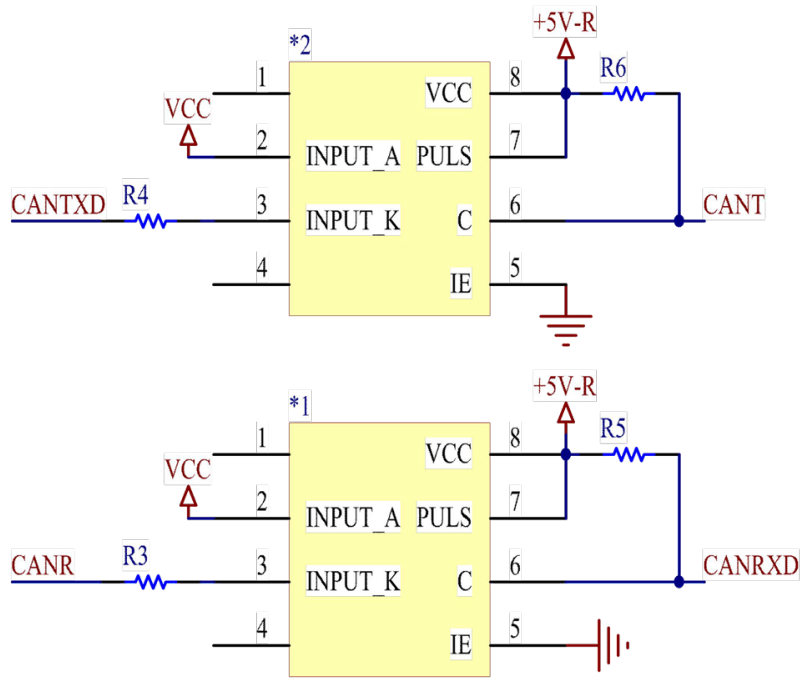

Figure 5 Optoelectronic isolation circuit

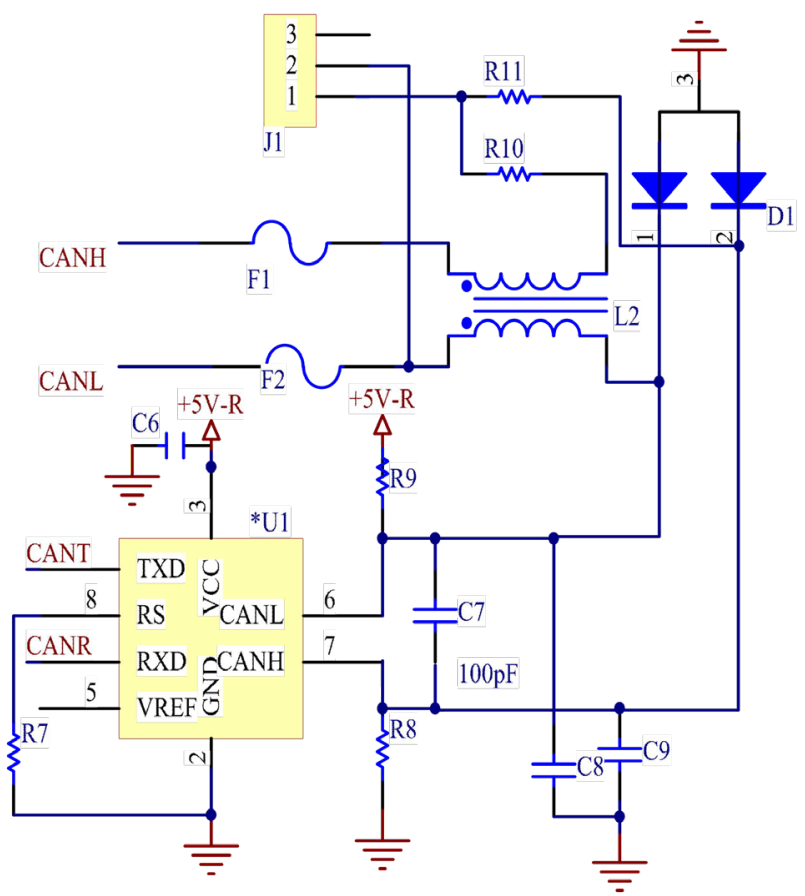

Figure 6 CAN Receiver PCA82C50 wiring diagram

\section{CAN communication protocol}

For CAN (Controller Area Network), it is a serial data communication protocol, with high performance, high reliability, easy to develop and low-cost field bus.It consists of CANH and CANL lines, the transmission medium can be unshielded twisted pair, cable and fiber, the communication rate up to $1 \mathrm{Mb} / \mathrm{S}$, and its transmission distance up to $10 \mathrm{Km}$. ${ }^{[5-8]}$

In this system, set up the control station, phase control rectifier, the inverter's own frame number.The control station sends the rectifier, the inverter frame number is 0X80 instruction frame, receives the real-time data of each electrical equipment and sends the control command signal.The frame number sent by the phase control rectifier to the control station is $180 \mathrm{H}$, and the 
frame numbers sent by the four inverters to the console are $181 \mathrm{H}, 182 \mathrm{H}, 183 \mathrm{H}$ and $184 \mathrm{H}$ respectively.As the end of the Table 1, 2, 3 is the byte definition of each module

\section{Experimental waveform}

For different devices and contactors connected to them, we will be 24 hours continuous monitoring, and the parameters of each device for real-time collection.By testing our system can run continuously for 24 hours without interruption. The following figure is our test in the CAN bus data waveform and CAN bus data frame waveform, as shown in Figure 7,8.

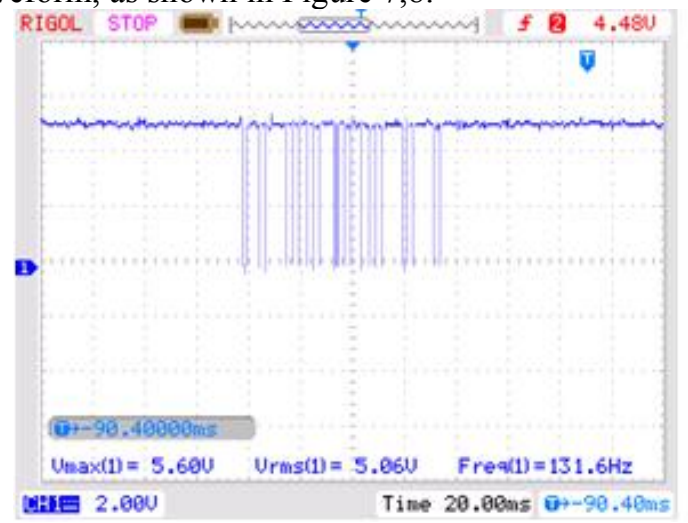

Figure 7 CAN bus data waveform

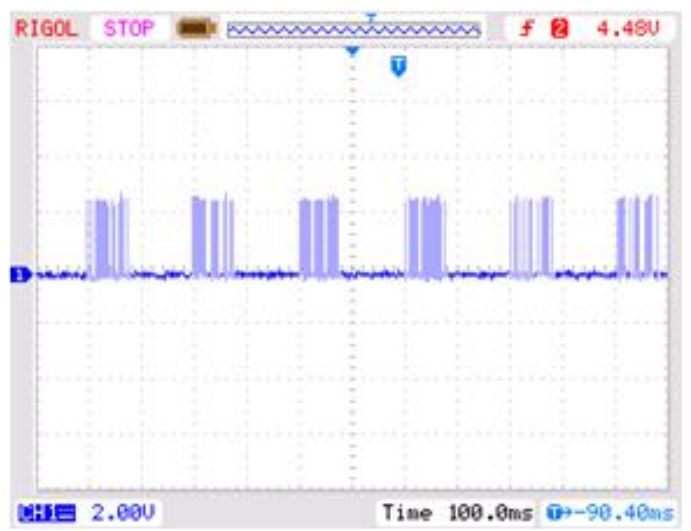

Figure $8 \mathrm{CAN}$ bus data frame waveform

After 24 hours of uninterrupted monitoring of the console display, we found that the four frequency converter through the CAN bus transmission voltage, current, frequency, fault and other information more timely and effective, and information feedback more stable and reliable.

\section{Conclusion}

Aiming at the auxiliary converter used in the locomotive, this paper designs the information transmission system which uses CAN bus technology for real-time communication. CAN bus system is a good solution to the locomotive power equipment operating frequency, current, voltage, fault and other information transmission problems, and managers can timely and accurate through the console display to get the operating parameters of each electrical equipment, with fast and accurate, high reliability characteristics. Through the installation and commissioning and operation of the locomotive, the system of seismic, electromagnetic compatibility, reliability and other aspects of the design can meet the practical requirements. This paper provides a reliable basis for the use of CAN bus technology in locomotives, and has good marketing value.

\section{Fund Project:}

Hebei Province Science and Technology Support Project (16274603,16214408)

Central University of basic scientific research business fees $(3142017045,3142013101,3142016022)$

\section{References}

[1] A.J. YUAN ,X.M. Tang, etc. A generalized software design technique for CAN fieldbus instrument [J]. Computer Engineering, 27 (2001), 144-146.

[2] G.J. Bao , M.M. Ji, L. Zhang, T.L. Wang . CAN bus technology, system implementation and development trend. Journal of Zhejiang University of Technology, Feb. (2003).

[3] Y.T. Rao, J.J Zou , Y.Y Zheng . Fieldbus CAN principle and application technology [M]. Beijing: Beijing University of Aeronautics and Astronautics Press, (2003).

[4]N. Li. MDK-based STM32 processor development and application [M]. Beijing: Beijing University of Aeronautics and Astronautics Press .(2008).

[5]F.C. Xiong. Automobile CAN bus communication technology research [D]. Wuhan University of Science and Technology .(2009).

[6]X. Hong,W.C. Kuang,Y.P. Xu.Marway. remote monitoring system based on CAN bus [J]. Microcomputer Information, 24 (2008) ,73-74,97.

[7] D.B. Liu , Y.J. Chen , H.W. Mao , ect. Remote monitoring system of coal mine underground substation based on CAN bus and Ethernet [f]. Industrial and mining automation, 35 (2009), 11-15.

[8] C.Y. Huang ,Y.H. Deng, Q.Z. Zhang . A new type of CAN bus dust monitoring system [J]. Coal Mine Safety, 43 (2012) ,95-97. 


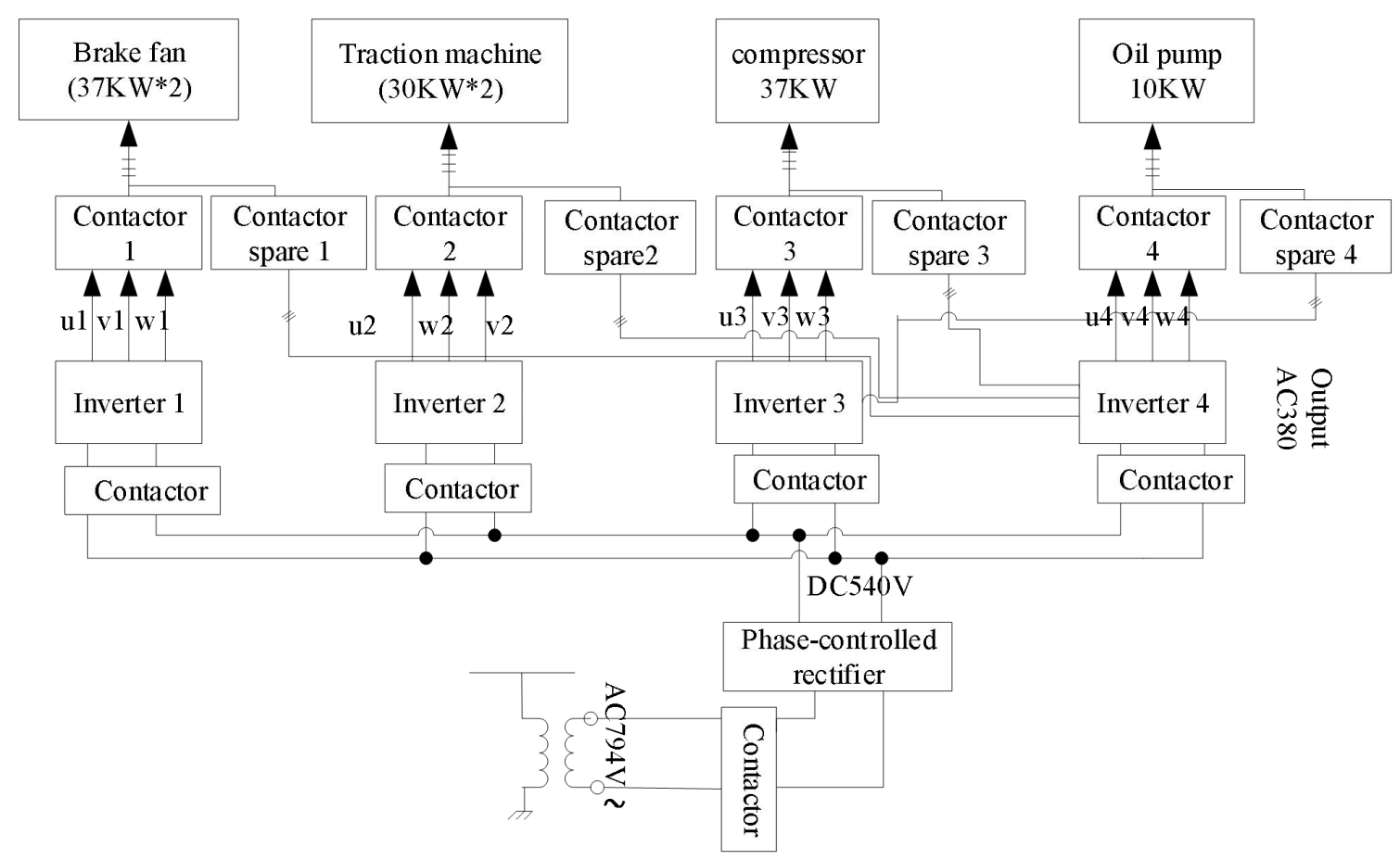

Figure 1 Auxiliary converter works

Table 1 Receive: Division console ID: 80

\begin{tabular}{|c|c|c|c|c|c|c|c|c|}
\hline & Bit7 & Bit6 & Bit5 & Bit4 & Bit3 & Bit2 & Bit1 & Bit0 \\
\hline Byte1 & \multicolumn{8}{|c|}{$80 \mathrm{H}$} \\
\hline Byte2 & \multicolumn{8}{|c|}{$08 \mathrm{H}$} \\
\hline Byte3 & \multicolumn{7}{|c|}{ inverter1 } & $\begin{array}{l}\text { run } 1, \\
\text { stop } 0\end{array}$ \\
\hline Byte4 & \multicolumn{7}{|c|}{ inverter2 } & $\begin{array}{l}\text { run 1, } \\
\text { stop 0 }\end{array}$ \\
\hline Byte5 & \multicolumn{7}{|c|}{ inverter3 } & $\begin{array}{l}\text { run 1, } \\
\text { stop 0 }\end{array}$ \\
\hline Byte6 & \multicolumn{7}{|c|}{ inverter4 } & $\begin{array}{l}\text { run } 1, \\
\text { stop } 0\end{array}$ \\
\hline Byte7 & \multicolumn{7}{|c|}{ rectifier } & $\begin{array}{l}\text { run 1, } \\
\text { stop 0 }\end{array}$ \\
\hline Byte8 & reserved & reserved & reserved & reserved & reserved & reserved & reserved & $\begin{array}{l}\text { normal } 0 \\
\text { failure } 1 \\
\end{array}$ \\
\hline
\end{tabular}

Table 2 Send: Rectifier ID: $180 \mathrm{H}$

\begin{tabular}{|c|c|c|c|c|c|c|c|c|}
\hline & Bit7 & Bit6 & Bit5 & Bit4 & Bit3 & Bit2 & Bit1 & Bit0 \\
\hline Byte1 & \multicolumn{8}{|c|}{$180 \mathrm{H}$} \\
\hline Byte2 & \multicolumn{8}{|c|}{$08 \mathrm{H}$} \\
\hline Byte3 & \multicolumn{6}{|c|}{ voltage low byte } & \multicolumn{2}{|c|}{$\begin{array}{c}\text { Normal } 0 \\
\text { overcurrent } 1\end{array}$} \\
\hline Byte4 & \multicolumn{8}{|c|}{ voltage high byte } \\
\hline Byte5 & \multicolumn{8}{|c|}{ current } \\
\hline Byte6 & \multicolumn{8}{|c|}{ temperature } \\
\hline Byte7 & \multicolumn{8}{|c|}{ rectifier } \\
\hline Byte8 & reserved & reserved & reserved & reserved & reserved & reserved & reserved & $\begin{array}{l}\text { normal } 0 \\
\text { failure } 1\end{array}$ \\
\hline
\end{tabular}

Table 3 Send: inverter1/2/3/4 ID: $181 \mathrm{H} / 182 \mathrm{H} / 183 \mathrm{H} / 184 \mathrm{H}$

inverter1 ID: $181 \mathrm{H}$




\begin{tabular}{|c|c|c|c|c|c|c|c|c|}
\hline & Bit7 & Bit6 & Bit5 & Bit4 & Bit3 & Bit2 & Bit1 & Bit0 \\
\hline Byte1 & \multicolumn{8}{|c|}{$181 \mathrm{H}$} \\
\hline Byte2 & \multicolumn{8}{|c|}{$08 \mathrm{H}$} \\
\hline Byte3 & \multicolumn{6}{|c|}{ voltage low byte } & \multicolumn{2}{|c|}{$\begin{array}{c}\text { normal } 0 \\
\text { overcurrent } 1\end{array}$} \\
\hline Byte4 & \multicolumn{8}{|c|}{ voltage high byte } \\
\hline Byte5 & \multicolumn{8}{|c|}{ current } \\
\hline Byte6 & \multicolumn{8}{|c|}{ temperature } \\
\hline Byte7 & \multicolumn{8}{|c|}{ rectifier } \\
\hline Byte8 & reserved & reserved & reserved & reserved & reserved & reserved & reserved & $\begin{array}{l}\text { normal } 0 \\
\text { failure } 1\end{array}$ \\
\hline
\end{tabular}

About the author: Li Yuanhong (1994-), male, Shanxi Yuncheng, undergraduate, graduate student of North China Institute of Science and Technology. Tel: 15732632524, E-mail: 15732632524@163.com 